Fernández, S.; Woitschach, P.; Álvarez-Díaz, M. y Fernández-Alonso, R. (2018). Análisis de la Oportunidad de Aprendizaje en el estudio TERCE de la UNESCO. Revista de Investigación Educativa, 36(2), 509-528.

DOI: http://dx.doi.org/10.6018/rie.36.2.307831

\title{
Análisis de la Oportunidad de Aprendizaje en el estudio TERCE de la UNESCO
}

\section{Analysis of the opportunity to learn in the TERCE study of UNESCO}

\footnotetext{
Samuel Fernández*, Pamela Woitschach** Marcos Álvarez-Díaz ${ }^{* * * *}$ y Rubén Fernández-Alonso

* Dpto. de Ciencias de la Educación de la Facultad de Formación del Profesorado y Educación. Universidad Oviedo (España)

* Dpto. de Metodología de la Facultad de Psicología. Universidad Complutense de Madrid (España)

* Servicio de Evaluación Educativa de la Consejería de Educación y Cultura del Principado de Asturias (España)

* Servicio de Evaluación Educativa de la Consejería de Educación y Cultura del Principado de Asturias (España) y Dpto. de Ciencias de la Educación de la Facultad de Formación del Profesorado y Educación. Universidad Oviedo (España)
}

\section{Resumen}

El objetivo de este estudio es analizar el efecto que la Oportunidad de Aprendizaje (OTL) tiene en el desempeño de las escuelas de América Latina, considerando los resultados obtenidos en la prueba de Ciencias Naturales del Tercer Estudio Regional Comparativo y Explicativo (TERCE) de las Naciones Unidas para la Educación la Ciencia y la Cultura (UNESCO). El estudio TERCE se realizó en el año 2013 en quince países de América Latina más el Estado de Nuevo León (México). La muestra estuvo compuesta por 61,937 estudiantes, con edad media de 12.42 años (DT=0.94). El 49.6\% son mujeres, el $69.4 \%$ asiste a un centro público, el $65,8 \%$ a un centro urbano y el $18.1 \%$ ha repetido un curso académico. Se ajustaron cuatro modelos jerárquicos lineales de intercepto aleatorios de tres niveles (alumno, escuela y país), empleando el programa HLM 7.01. Entre los principales resultados se observa que, una vez descontadas las variables socioeconómicas y el historial de repetición académica, los factores asociados al clima, a las prácticas de aula, y al desarrollo docente, en tanto que variables de OTL, presentan un margen de mejora destacable en el funcionamiento de las escuelas.

Palabras clave: oportunidad de aprendizaje; eficacia docente; ciencias naturales; modelos jerárquicos lineales.

Correspondencia: Pamela Woitschach pamelawo@ucm.es, Dpto. de Metodología de la Facultad de Psicología. Universidad Complutense de Madrid (España). 


\begin{abstract}
The purpose of this study is to analyse the effect of Opportunity To Learn (OTL) on the performance of schools in Latin America, considering the results obtained in the Natural Sciences test of the Tercer Estudio Regional Comparativo y Explicativo (TERCE) (Third Regional Comparative and Explanatory Study of the United Nations Educational, Scientific and Cultural Organization (UNESCO). TERCE was carried out in 2013 in fifteen countries in Latin America and in the State of Nuevo Leon (Mexico). The sample was 61,937 students, with a mean age of 12.42 years $(S D=0.94) .49 .6 \%$ are women, $69.4 \%$ attend a public school, $65.8 \%$ are allocated in an urban school and $18.1 \%$ have repeated an academic course. Four linear hierarchical models of three-level random intercepts (student, school and country) were applied. The HLM7.01 program was used. It can be observed that, among the main results, once the socioeconomic variables and the academic repetition background are discounted, the factors related to: climate, teaching practices, and teacher development-as OTL variables- have a positive remarkable effect on school performances.

Keywords: opportunity to learn; instructional effectiveness, science achievement; hierarchical linear models.
\end{abstract}

\title{
Introducción
}

El concepto de Oportunidad de aprendizaje (OTL), cuyo origen está en el modelo de Carroll (1963) que lo definió como la cantidad de tiempo determinado para aprender, ha sido ampliamente tratado en la investigación educativa por ser considerado un factor clave a la hora de explicar las diferencias de rendimiento del alumnado y su estudio ha modificado en los últimos años la visión sobre los determinantes del rendimiento educativo (McDonnell, 1995). El metanálisis de Scheerens (2017), que incluye estudios realizados entre 1997 y 2009, reporta un tamaño del efecto $(d)$ de las variables OTL de 0.44 , superior al efecto de factores como la implicación familiar o el liderazgo escolar. Lamain, Scheerens y Noort (2017) recopilan 51 estudios primarios realizados en las dos últimas décadas en todas las regiones mundiales y contabilizan que el tamaño del efecto de las variables OTL es positivo y significativo en 84 de los 192 efectos analizados, es decir, en el $44 \%$ de los casos recopilados.

Las evaluaciones internacionales (v. g., el Estudio Internacional de Tendencias en Matemáticas y Ciencias -TIMSS o el Programa para la Evaluación Internacional de Alumnos -PISA) han mostrado el efecto de las variables OTL sobre el rendimiento. Angell, Kjærnsli y Lie (2006), con datos de TIMSS, destacan que el grado de cobertura de los contenidos curriculares explica entre el 25\% y el 50\% de la varianza entre los países. Klieme (2016) estima que la medida OTL en TIMSS 2011 incrementa el poder explicativo de las diferencias entre países entre un 3 y un 6\%, y en el caso de PISA 2012 la varianza entre países pasa del $85 \%$ al $96 \%$ al incluir la OTL como predictor. Mo, Singh y Chang (2013) reanalizando datos de TIMSS 2003 encuentran una asociación positiva entre el resultado en Ciencias y las variables OTL. Por otra parte, Luyten (2017) concluye que el efecto de variables OTL sobre el rendimiento científico-matemático es positivo y alto en los 22 países de PISA 2012 analizados, y relativamente moderado en TIMSS 2011, resultado tal vez ligado a la validez de la medida OTL. Como segunda derivada de las 
comparaciones internacionales, las variables OTL, además de explicar diferencias en el rendimiento, pueden ayudar a desarrollar modelos de enseñanza-aprendizaje más adaptados a los curricula nacionales, sin necesidad de remarcar puntajes diferenciales entre realidades socioculturales diversas (Floden, 2002).

Por su parte, los estudios de eficacia escolar en Latinoamérica han señalado que, el efecto positivo de la OTL sobre los resultados escolares se mantiene incluso después de controlar las variables de contexto (Cueto, Guerrero, León, Zapata \& Freire 2014; Cueto, León, Ramírez \& Guerrero, 2008; Fernández, 2004; Martínez-Garrido \& Murillo, 2016; Murillo, 2007; Murillo \& Hernández-Castilla, 2011; UNESCO-OREALC \& LLECE, 2000, 2010; Velez, Schiefelbein \& Valenzuela, 1994).

Hasta el momento el término OTL se ha tratado como un constructo unidimensional, cuando en realidad está lejos de tener una acepción unívoca. Durante las últimas décadas el significado original (tiempo de aprendizaje) se ha complejizado y ahora se considera un concepto poliédrico y multimensional, que exige el uso de diversos métodos de recogida de información y el manejo de diferentes variables. Para su estimación los estudios con grandes muestras emplean mayoritariamente métodos indirectos (encuestas al alumnado y profesorado), aunque también son posibles procedimientos directos (observación de aula o revisión de las tareas y cuadernos del alumnado). Igualmente las variables empleadas son diversas, si bien las más frecuentes son: currículum impartido y tasas de cobertura del contenido; tiempo de aprendizaje; calidad de la instrucción, de las prácticas de enseñanza y de los recursos; y gestión, disciplina, clima de trabajo y relaciones personales en el aula (Cueto et al., 2008, 2014; OECD, 2013). A continuación se revisan algunos estudios previos que han manejado variables OTL similares a las disponibles en la base de datos del Tercer Estudio Regional Comparativo y Explicativo (TERCE), que será la matriz empleada en el presente análisis. Se trata de variables vinculadas al tiempo de aprendizaje, las características del proceso instructivo, los recursos disponibles y el ambiente de trabajo del aula.

Como ya se señaló, el tiempo de aprendizaje es un elemento canónico del constructo OTL (Fernández, Fernández-Alonso, Arias, Fernández-Raigoso \& Burguera, 2016). Stallings, Knight y Markham (2014) estiman que, en un aula óptimamente gestionada, al menos el $85 \%$ del tiempo se dedica a actividades de enseñanza y aprendizaje y el 15\% a tareas administrativas, logísticas y de gestión. Bruns y Luque (2014) encuentran que los países iberoamericanos quedan 20 puntos porcentuales por debajo de ese $85 \%$, lo que se traduce en un día menos de instrucción por semana. Observaciones estandarizadas en el aula han mostrado que la pérdida significativa del tiempo de instrucción es un fenómeno importante en Latinoamérica, siendo el absentismo docente una de las principales causas, ya que la mitad del tiempo perdido se debe a ausencias del profesorado, impuntualidad al inicio o finalización de las clases o a la realización durante las mismas de actividades distintas de las instructivas (Abadzi, 2009; Chaudhury, Hammer, Kremer, Muralidharan \& Rogers, 2006). Los datos anteriores pueden incluso considerarse conservadores, al menos comparados con la estimación de Wolff, Schiefelbein y Valenzuela (1994) que señalaban que en la región se perdía un tercio del calendario lectivo por huelgas, y ausencias docentes. En todo caso, los resultados disponibles muestran que el tiempo sobre la tarea está positivamente asociado con el rendimiento en pruebas estandarizadas en diferentes 
contextos (rural o urbano, bilingues o monolingues), edades y materias (Cueto \& Secada, 2003; Hernández-Castilla, Murillo \& Martínez-Garrido, 2014; Schuh Moore, DeStefano \& Adelman, 2012). Los estudios del Laboratorio Latinoamericano de la Calidad Educativa (LLECE) señalan que la asistencia y puntualidad docente aumentan el promedio de los estudiantes entre 6 y 34 puntos, según el país y la materia, encontrándose que este efecto significativo se reproduce en todos los países, aún después de descontar el efecto del nivel socioeconómico del alumnado, y similares resultados también se encontraron con muestras españolas (Servicio de Evaluación Educativa del Principado de Asturias, 2017). No obstante, los resultados no son unánimes. Así, Velez et al. (1994), después de revisar 18 estudios latinoamericanos sobre el tema, contabilizaron 60 efectos distintos que describen la relación absentismo docenterendimiento académico de los cuales sólo 18 confirmaban la hipótesis de que a mayor absentismo peores resultados, mientras que en 34 casos la relación, si bien positiva, no era significativa. Carvallo (2006), con datos del estudio del Examen Nacional de Ingreso (EXANI) de México, también señala un resultado atípico, no pudiendo asociar la impuntualidad docente con descensos en los rendimientos escolares.

Un segundo elemento del constructo OTL es el clima de aula, entendido como la atmósfera de orden, buenas relaciones y ambiente de trabajo orientado a la consecución de los objetivos educativos (Scheerens, 2016). La información disponible sobre la relación entre clima de aula y resultados educativos ofrece un panorama consistente y coherente. Marzano, Marzano y Pickering (2003) estiman que el orden de aula tiene un efecto moderado $(\mathrm{d}=.52)$ sobre el rendimiento académico, si bien con los datos de Korpershoek, Harms, de Boer, van Kuijk, y Doolaard (2016) este efecto se puede calificar de pequeño aunque significativo $(g=.17)$. A medio camino entre ambas estimaciones se encuentran Durlak, Weissberg, Dymnicki, Taylor y Schellinger (2011), que obtienen un efecto entre pequeño y moderado $(g=.27)$. Los datos de PISA señalan que las diferencias en Matemáticas según la percepción discente del orden de aula son del 40\% de la desviación típica para el conjunto de la OCDE, confirmándose esta relación, en mayor o menor medida, en todos los países (Gil Flores, 2014; Gobierno del Principado de Asturias, 2011). En el contexto latinomericano las tres evaluaciones del LLECE apuntan en la misma dirección: en el primer estudio regional (PERCE) el clima de aula predecía diferencias en Matemáticas y Lectura en torno a una desviación típica, mientras que en el segundo (SERCE) las ganancias esperadas eran más moderadas (entre 10 y 60 puntos según la materia y el país). Finalmente, en TERCE el rango de las diferencias en función del clima de aula osciló entre 6 y 18 puntos (UNESCO-OREALC \& LLECE, 2016a). De igual modo, existe un buen número de investigaciones que señalan que las aulas con buenas relaciones personales y un clima de trabajo estimulante, cordial, afectivo y seguro tienden a presentar mejores resultados educativos (Carvallo, 2006; López, 2006; Ramos-Ramírez, 2013; Román, 2010; Torres-Fernández, 2008). En esta misma línea argumental se ha encontrado que en las escuelas de bajo rendimiento el clima de aula se caracteriza por la tensión, la escasa participación y las malas relaciones entre docentes y discentes (Hernández-Castilla et al., 2014). Finalmente, algunos datos apuntan a que en las aulas con mejor clima de trabajo los estudiantes también presentan ganancias en factores socioafectivos (motivación, autoconcepto) y mayor satisfacción hacia la escuela (Martínez-Garrido, 2015). 
Un tercer elemento que configura el constructo OTL son las prácticas de enseñanza, que incluyen el modo de trabajar los contenidos de aprendizaje, la metodología didáctica, las explicaciones, el tipo, orginización y orientación de las tareas de enseñanza y la evaluación de los aprendizajes. Se ha encontrado una relación positiva entre los resultados educativos y ciertas tareas docentes como establecer objetivos de aprendizaje claros, plantear actividades de alto nivel de exigencia cognitiva, controlar el trabajo y los deberes en el hogar y organizar una evaluación variada y justa (Gobierno del Principado de Asturias, 2011), y análisis complementarios sugieren que el alumnado con niveles de comprensión más bajos se beneficia especialmente cuando asiste a un aula en la que la claridad de las explicaciones del profesorado es valorada positivamente por la mayoría del alumnado, en tanto que el alumnado con mayor nivel de comprensión se beneficia cuando se le enfrenta a actividades de aprendizaje con alta exigencia cognitiva y que demandan reflexión sobre el propio aprendizaje, lo cual señala la necesidad de establecer prácticas de enseñanza diferenciadas y de claro nivel reflexivo e investigador (Fernández et al., 2016). La investigación latinoamericana apunta a conclusiones similares. Si bien los primeros estudios del LLECE no encontraron resultados concluyentes (UNESCO-OREALC \& LLECE, 2000, 2010), en cambio TERCE estima que las prácticas docentes predicen ganancias de entre 9 y 36 puntos en las pruebas cognitivas (UNESCO-OREALC \& LLECE, 2016a). Igualmente, Martínez-Garrido (2015) y Román (2010) encuentran efectos positivos de la metodología docente sobre los resultados. En conjunto, los datos asocian ciertas características docentes a buenos resultados, tales como clases bien preparadas, enseñanza estructurada, objetivos claros, colaboración docente, actividades variadas y participativas, estrategias de aprendizaje activas y uso frecuente de la evaluación y seguimiento del progreso del alumnado (Murillo \& Román, 2009; Torres-Fernández, 2008; Velez et al., 1994). Por el contrario, las escuelas tienden a presentar rendimientos más bajos cuando la metodología docente se basa en estrategias de memorización y reproducción de contenidos (Carvallo, 2006; Hernández-Castilla et al., 2014).

El último componente del concepto OTL son los recursos materiales disponibles. Los resultados de la investigación parecen dependientes del contexto en el que se desarrollan los estudios. Así, Gaviria, Martínez-Arias y Castro (2004) han señalado que en los países desarrollados, donde el gasto educativo es razonablemente alto y las diferencias en la calidad y cantidad de los recursos de las escuelas son relativamente bajas, las evidencias sobre la relación recursos-resultados tiende a ser débil, pero cuando estos estudios se realizan en países en vías de desarrollo el efecto de los recursos sobre el rendimiento es más nítido. En el contexto latinoamericano, Velez et al. (1994), después de analizar varios informes de investigación del último cuarto del siglo XX, encontraron una relación positiva entre la disponibilidad de libros de texto y materiales de lectura y el rendimiento en 13 de las 17 investigaciones revisadas, y Gaviria et al. (2004) concluyen que los recursos de la escuela impactan en los resultados educativos, haciendo mención especial a la existencia o no de libros de texto. Por su parte, Murillo y Román (2009), analizando los datos de SERCE, indican que la existencia de recursos didácticos y las instalaciones académicas constituyen el segundo factor escolar con mayor efecto sobre el desempeño de los estudiantes. En una línea similar los datos de TERCE señalan que el simple hecho de disponer de un cuaderno 
individual de trabajo como recurso de uso habitual en el aula, se relaciona con un mejor rendimiento en la mitad de la muestra de tercer grado, y que el $17.6 \%$ de los estudiantes de este nivel no cuenta con el material de estudio apropiado, así como el 28.9\% de los estudiantes de sexto grado, lo que refleja que, a pesar de que el nivel de pobreza ha disminuido en los últimos años, aún se carece de recursos básicos en el aula (UNESCO-OREALC \& LLECE, 2016a).

\section{Método}

La estructura jerárquica de la información de las evaluaciones educativas internacionales y la búsqueda de modelos que ajusten las variables contextuales de cada nivel a la variabilidad individual de rendimiento escolar reclama el uso de una metodología que permita analizar la interacción entre factores individuales y sociales.

\section{Objetivos}

La finalidad del presente trabajo es analizar el impacto que tiene la oportunidad de aprendizaje (OTL) en los resultados del alumnado latinoamericano de sexto grado de educación en Ciencias Naturales. Este objetivo general se concreta en las siguientes cuestiones:

1. ¿Qué impacto tiene sobre los resultados en Ciencias Naturales la asistencia y metodología docente, el clima de trabajo en el aula y la disponibilidad por parte del alumnado de un recurso básico como el cuaderno de trabajo?

2. Una vez controladas las variables antecedentes del contexto educativo, ¿se sigue manteniendo este hipotético impacto de las variables relacionadas con la oportunidad de aprendizaje?

3. ¿Qué porcentaje de varianza de los resultados educativos explican las variables asociadas a la oportunidad de aprendizaje?

\section{Población y Muestra}

La población se definió como el alumnado matriculado en sexto curso de enseñanza obligatoria en el curso 2013 en los 15 países participantes y en el estado de Nuevo León (México). En cada país la muestra fue seleccionada siguiendo un diseño bietápico por conglomerados y estratificado propio de las evaluaciones internacionales (Joncas \& Foy, 2012; OECD, 2009). En la primera etapa los centros (unidades primarias de la muestra) fueron seleccionados con una probabilidad proporcional a su tamaño, y en la segunda etapa se seleccionó un grupo-aula completo de cada centro, obteniéndose una muestra por encima de los 67.000 estudiantes. Del presente estudio se excluyó al alumnado sin información en la prueba de Ciencias Naturales, por lo que la base final quedó compuesta por 61.637 estudiantes escolarizados en 2.955 centros educativos, que representan a una población de prácticamente 9 millones de estudiantes escolarizados en sexto curso en la región. La Tabla 1 recoge el número de estudiantes participantes y la población total a la que representan por país. 
La media de edad del alumnado es de 12.42 años y la desviación típica 0.94 . El $69.4 \%$ asiste a un centro público y el $65.8 \%$ a un centro urbano; el $49.6 \%$ son mujeres y el $81.9 \%$ está escolarizado en el curso correspondiente a su edad en tanto que el $18.1 \%$ restante ha repetido al menos un curso en el momento de la aplicación de la prueba.

Tabla 1

Datos de la muestra y la población

\begin{tabular}{lrr}
\hline & Tamaño de la muestra & Tamaño de la población \\
\hline Argentina & 3,639 & 760,311 \\
Brasil & 2,983 & $2,043,907$ \\
Chile & 5,044 & 262,569 \\
Colombia & 4,308 & $1,046,752$ \\
Costa Rica & 3,520 & 105,218 \\
República Dominicana & 3,661 & 184,352 \\
Ecuador & 4,818 & 416,114 \\
Guatemala & 4,056 & 227,627 \\
Honduras & 3,880 & 170,860 \\
México & 3,618 & $2,599,591$ \\
Nicaragua & 3,726 & 115,937 \\
Panamá & 3,413 & 66,069 \\
Paraguay & 3,222 & 118,744 \\
Perú & 4,789 & 609,457 \\
Uruguay & 2,799 & 52,096 \\
Nuevo León (México) & 4,197 & 115,783 \\
Total & 61,673 & $8,895,387$ \\
\hline
\end{tabular}

\section{Instrumentos}

En el estudio se emplearon dos tipos de instrumentos: (a) pruebas de conocimientos escolares, a partir de las cuales se construye la variable dependiente del estudio; (b) cuestionarios de contexto para el alumnado, sus familias, el profesorado y las direcciones de los centros, de donde se extraen todas las variables de interés y de ajuste del presente estudio, salvo la relativa al nivel de riqueza de los países. Las pruebas fueron aplicadas en el programa de evaluación TERCE de las Naciones Unidas para la Educación la Ciencia y la Cultura (UNESCO), cuyas bases de datos son de acceso libre para su uso en investigación (UNESCO-OREALC, 2016).

\section{Rendimiento en Ciencias Naturales}

El alumnado respondió a una batería de pruebas que evaluaban Lectura, Matemáticas y Ciencias, si bien en el presente estudio se decidió usar los resultados de 
Ciencias como variable dependiente. La prueba de Ciencias se desarrolló a partir de una tabla de especificaciones organizada en cinco dominios y tres procesos cognitivos (UNESCO-OREALC, 2016) y constaba de 92 ítems, en su mayoría de elección múltiple, agrupados en seis bloques (cuatro bloques de ítems nuevos y dos bloques con ítems de anclaje provenientes de SERCE). Los ítems se distribuyeron en seis cuadernillos siguiendo un diseño matricial (Fernández-Alonso \& Muñiz, 2011), y cada estudiante respondió a un cuadernillo que contenía entre 31 y 33 ítems a resolver en unos 60 minutos de evaluación. Los ítems fueron ajustados al modelo Rasch empleando el programa Winsteps (Linacre, 2005). La puntuación de cada estudiante fue calculada mediante la metodología de valores plausibles que es la más eficiente para recuperar los parámetros poblaciones en las evaluaciones de sistemas educativos (Mislevy, Beaton, Kaplan, \& Sheehan, 1992; OECD, 2009; von Davier, Gonzalez, \& Mislevy, 2009). En TERCE, las puntuaciones individuales se estimaron conjugando las respuestas de los estudiantes a los ítems con información proveniente de diferentes covariables que funcionan como factores de imputación, y fueron expresadas en una escala con media de 700 puntos y desviación típica 100 (UNESCO-OREALC \& LLECE, 2016b).

\section{Variables de ajuste}

Cuando la variable dependiente es el rendimiento escolar conviene incluir variables de ajuste que eviten sobreestimar los efectos de las variables de interés (Fernández-Alonso, Álvarez-Díaz, Suárez-Álvarez \& Muñiz, 2017). Dentro de los datos disponibles en la matriz de TERCE se han elegido seis variables de control que permiten describir las características sociológicas del alumnado. Se trata de variables tradicionalmente relevantes en la predicción de rendimiento académico (Liu \& Whitford, 2011; UNESCO-OREALC \& LLECE, 2016a; Woitschach, FernándezAlonso, Martínez-Arias \& Muñiz, 2017). Cinco de ellas son dicotómicas: Género (1 = ser mujer); condición de Indígena ( 1 = pertenecer a una etnia indígena); condición de Repetición ( 1 = haber repetido algún curso durante la escolaridad); Trabajo remunerado (1= el estudiante trabaja y recibe una remuneración por esa actividad); y Conexión a Internet $(1=$ el estudiante dispone de conexión a Internet en el hogar). La última variable es una estimación del Nivel Socioeconómico y Cultural del alumnado (SEC), que es un índice estandarizado construido por TERCE y compuesto por 17 ítems que recogen información sobre el nivel educativo de los padres, el tipo de trabajo que realizan, el rango de ingresos familiares, así como información sobre los bienes y servicios del barrio en el que se ubica la vivienda, y la disponibilidad de material de lectura del hogar. Los valores del alfa de Cronbach de este índice oscilan entre 8 y .9 según el país (UNESCO-OREALC, 2016).

Dentro de las características del contexto social y demográfico de la escuela se han considerado cuatro variables, dos de ellas dicotómicas: Titularidad ( 1 = centro privado) y Ruralidad del centro ( 1 = centro rural). El volumen de recursos de la escuela se estimó mediante el Nivel de Infraestructura de la escuela, que es un índice estandarizado elaborado con información de 10 ítems del cuestionario del director referidos al tipo de instalaciones, equipamientos y servicios con los que cuenta la escuela. Los valores del 
alfa de Cronbach de este índice oscilan entre .7 y .9 según el país (UNESCO-OREALC, 2016). La cuarta variable es el Nivel Socioeconómico y Cultural de la escuela, estimado como el promedio por centro del SEC del alumnado escolarizado en el mismo.

Finalmente, se ha considerado una variable de ajuste a nivel de país, en este caso una estimación del nivel de riqueza, medido a través del Producto Interno Bruto Per Cápita del año 2013 (UNESCO-OREALC \& LLECE, 2016a).

\section{Variables de interés (OTL)}

En total se han manejado cuatro variables OTL, dos de ellas a nivel de alumnado: Cuaderno ( $1=$ el estudiante tiene cuaderno o libreta para tomar notas en clase) y un índice estandarizado que estima la Asistencia y Puntualidad Docente, y que fue construido con las respuestas del alumnado a tres ítems de elección múltiple: el profesorado falta a clases, llega tarde y se va temprano, y cuyas opciones de respuesta eran: $1=$ "Nunca o casi nunca"; 2 = "A veces"; 3 = "Siempre o casi siempre". El valor del alfa de Cronbach oscila entre .4 y .7, según el país (UNESCO-OREALC, 2016).

Se han manejado además dos variables, expresadas como índices estandarizados a nivel de centro: el índice Prácticas para el Desarrollo del Aprendizaje, elaborado a partir de la respuesta del alumnado a 13 ítems que se refieren a la frecuencia con la que ocurren ciertos hechos en la clase (por ejemplo, el profesorado nos motiva a seguir estudiando, tienen las clases preparadas, etc.). Las opciones de respuesta eran: $1=$ "Nunca o casi nunca"; 2 = "A veces"; 3 = "Siempre o casi siempre". El valor del alfa de Cronbach de este índice oscila entre .6 y .9 según el país (UNESCO-OREALC, 2016). Por su parte, el índice Clima de Aula se construyó con las respuestas a seis ítems destinados a valorar el nivel de atención del alumnado en el aula, el respeto entre el alumnado, el número de interrupciones durante las clases o la frecuencia de comportamientos agresivos. El alfa de Cronbach para este índice osciló entre .7 y .9 (UNESCO-OREALC, 2016, p. 303).

\section{Procedimiento de recogida y análisis de datos}

La aplicación de las pruebas cognitivas fue realizada por personal experto y externo al centro y se desarrolló en dos jornadas, la primera dedicada a Lectura y Escritura y la segunda a Matemáticas y Ciencias Naturales. La evaluación de cada materia ocupó entre 45 y 60 minutos, con un descanso de 30 minutos; la aplicación de los cuestionarios de contexto del alumnado tuvo una duración de 45 minutos, tras un receso de 15 minutos. El primer día se entregaron los cuestionarios para el centro, profesorado y familias, que se recogieron al final de la segunda jornada. Se tuvieron en cuenta los estándares éticos de la UNESCO, y las familias de los estudiantes seleccionados recibieron una comunicación a través de las direcciones escolares sobre su participación en el estudio.

En el análisis de datos inicialmente se calcularon los estadísticos descriptivos de todas las variables y las correlaciones de Pearson. A continuación, se ajustaron cuatro modelos jerárquico-lineales de interceptos aleatorios y segmentados en tres niveles: alumno, centro y país. La estrategia de modelización fue la siguiente: inicialmente, se ajustó un modelo nulo sin predictores, para comprobar la distribución de la varianza en cada nivel. El segundo modelo incluyó sólo las variables de ajuste, el tercero sólo 
las variables OTL y el último consideró conjuntamente las variables de los modelos previos. En el ajuste se empleó el método de estimación de máxima verosimilitud con errores típicos robustos usando el Programa HLM 7.01 (Raudenbush, Bryk, Cheong, Congdon \& du Toit, 2011). En todos los análisis se utilizaron los pesos senatoriales provistos por TERCE, los cuales están diseñados para que todos los países, independientemente del tamaño de su población, contribuyan por igual en el análisis de resultados (UNESCO-OREALC, 2016). En TERCE la suma total de los pesos de cada país equivale a 5000 estudiantes (peso del nivel 1) escolarizados en 200 escuelas (peso del nivel 2).

El rango de casos perdidos en las variables osciló entre el 2\% y el 12\%, y para su recuperación se empleó una estrategia en dos pasos. Inicialmente los casos incompletos fueron imputados con la media del sujeto y, a continuación, los datos totalmente perdidos se recuperaron mediante el método iterativo EM con variables auxiliares que implementa el módulo Missing Value Análisis de SPSS 22. Fernández-Alonso, Suárez-Álvarez y Muñiz (2012) encontraron que esta estrategia en dos pasos es la que mejor recupera los datos poblacionales en estudios con tipos de pérdida (no aleatoria) y porcentajes de datos faltantes similares a los registrados en TERCE.

\section{Resultados}

La Tabla 2 muestra los estadísticos descriptivos y correlaciones entre las variables. En general, el sentido de las asociaciones es el esperado: las aulas con mejor clima tienden también a presentar mejores puntuaciones en las prácticas educativas $(r=.23)$ y el profesorado con mejores valores en el índice de asistencia docente, también presenta mejores puntuaciones en el índice de prácticas educativas $(r=.15)$. Igualmente, las correlaciones entre las variables independientes y el rendimiento en Ciencias Naturales presentan, en general, la magnitud y dirección esperadas.

La Tabla 3 muestra los resultados obtenidos en el ajuste multinivel, con las variables organizadas por cada nivel de análisis. El modelo nulo indica que la mayor parte de la varianza (54\%) se sitúa dentro de los centros, en tanto que, prácticamente uno de cada tres puntos de la varianza se ubica en el nivel entre centros. Finalmente, el 14\% de las diferencias se encuentran entre los países.

El modelo I, que incluye todas las variables de ajuste, explica el 20\% de la diferencia total y una parte importante de las diferencias entre los centros. Todas las variables de ajuste a nivel de centro son significativas y operan en la dirección esperada. Destaca, en todo caso, el efecto del SEC de centro, ya que por cada punto que aumenta se predice ganancias cercanas al 35\% de la desviación típica en la escala de Ciencias. En el nivel individual la variable con más impacto es la repetición de curso, en el sentido de que al alumnado repetidor se le predice una pérdida cercana a un cuarto de desviación típica en la escala de Ciencias.

El modelo II, sólo con variables OTL, explica el 5\% de la varianza total y casi el 10\% de las diferencias entre centros. De nuevo todas las variables funcionan en la dirección esperada. Cabe destacar que en este modelo el índice de prácticas docentes parece tener más fuerza explicativa que el clima de aula.

El Modelo III, que incluye todas las variables, explica prácticamente el $25 \%$ de la varianza total y casi la mitad de las diferencias entre los centros. Todas las variables de 


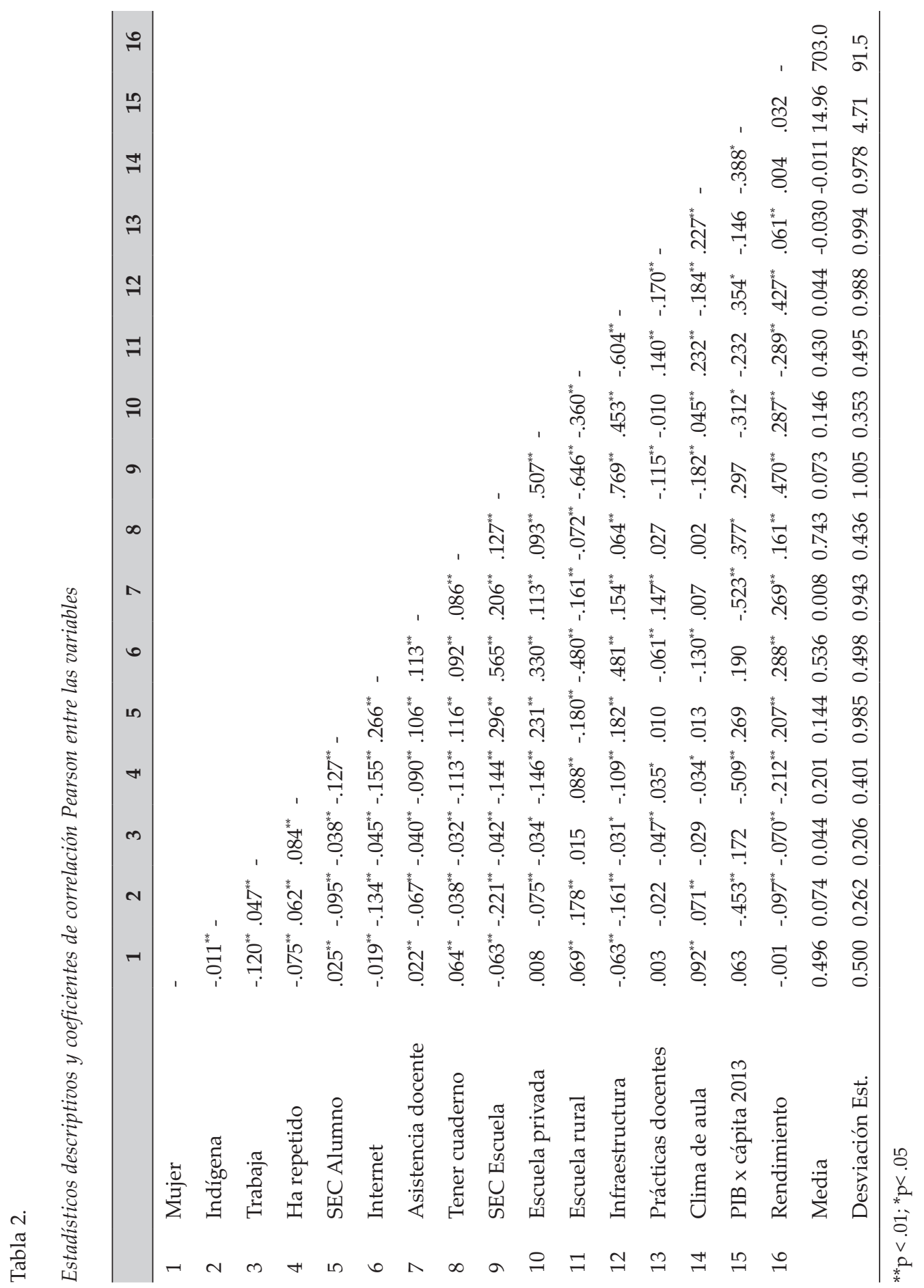


interés continúan siendo significativas pese a estar controladas por las variables de ajuste $\mathrm{y}$, de hecho, el efecto de las variables de interés apenas pierde fuerza explicativa. Por su parte la significación de las variables de ajuste también funciona en el sentido esperado.

Tabla 3

Modelos Multinivel para predecir el efecto de la oportunidad de aprendizaje en Ciencias Naturales (estudio TERCE)

\begin{tabular}{|c|c|c|c|c|}
\hline & $\begin{array}{c}\text { Modelo } \\
\text { Nulo } \\
\beta\end{array}$ & $\begin{array}{c}\text { Modelo I } \\
\text { Contexto } \\
\beta(\mathrm{SE})\end{array}$ & $\begin{array}{l}\text { Modelo II: } \\
\text { OTL } \\
\beta(\mathrm{SE})\end{array}$ & $\begin{array}{c}\text { Modelo III: } \\
\text { Contexto+OTL } \\
\beta(\mathrm{SE})\end{array}$ \\
\hline Intercepto & $696.27(9.66)^{* * *}$ & $636.61(23.14)^{* * * *}$ & $687.79(9.57)^{* * *}$ & $640.15(20.01)^{* * *}$ \\
\hline \multicolumn{5}{|l|}{ Nivel 1 (Alumnado) } \\
\hline Mujer & - & $-1.96(1.96)$ & - & $-3.02(2.01)$ \\
\hline Indígena & - & $-0.01(3.58)$ & - & $1.93(3.88)$ \\
\hline Trabaja & - & $-10.73(6.46)$ & - & $-9.33(5.66)$ \\
\hline Ha repetido & - & $-22.51(5.53)^{* * * *}$ & - & $-20.92(2.81)^{* * *}$ \\
\hline SEC Alumno & - & $5.95(2.01)^{* *}$ & - & $5.66(1.55)^{* * *}$ \\
\hline Internet/casa & - & $8.31(3.44)^{*}$ & - & $7.48(2.95)^{*}$ \\
\hline Asistencia docente & - & - & $12.40(1.72)^{* * *}$ & $11.21(1.12)^{* * *}$ \\
\hline Tener cuaderno & - & - & $13.10(2.97)^{* * *}$ & $11.04(2.55)^{* * *}$ \\
\hline \multicolumn{5}{|l|}{ Nivel 2 (Centro) } \\
\hline SEC Escuela & - & $34.94(4.82)^{* * *}$ & - & $32.93(3.90)^{* * *}$ \\
\hline Escuela privada & - & $14.30(6.14)^{*}$ & - & $10.09(5.60)^{+}$ \\
\hline Escuela rural & - & $21.84(11.43)^{\dagger}$ & - & $18.28(5.47)^{* * *}$ \\
\hline Infraestructura & - & $7.81(3.55)^{* *}$ & - & $9.42(3.12)^{* *}$ \\
\hline Prácticas docentes & - & - & $8.23(3.69)^{*}$ & $10.28(2.14)^{* * *}$ \\
\hline Clima de aula & - & - & $6.14(2.35)^{* *}$ & $6.22(2.23)^{* *}$ \\
\hline \multicolumn{5}{|l|}{ Nivel 3 (País) } \\
\hline PIB x Cápita 2013 (mil. \$) & - & $0.79(0.77)$ & - & $0.79(1.12)$ \\
\hline \multicolumn{5}{|l|}{ Distribución de la varianza } \\
\hline Dentro del centro & 5903.34 & 5783.13 & 5786.84 & 5682.59 \\
\hline Entre los Centros & 3560.36 & 2196.31 & 3225.10 & 1999.65 \\
\hline Entre los Países & 1465.71 & 745 & 1414.25 & 645.71 \\
\hline Total & 10929.41 & 8724.44 & 10426.19 & 8327.95 \\
\hline \multicolumn{5}{|l|}{ Porcentaje de varianza explicada } \\
\hline Dentro del Centro & - & $2 \%$ & $2 \%$ & $4 \%$ \\
\hline Entre los Centros & - & $38 \%$ & $9 \%$ & $44 \%$ \\
\hline Entre los Países & - & $49 \%$ & $4 \%$ & $56 \%$ \\
\hline Total & - & $20 \%$ & $5 \%$ & $24 \%$ \\
\hline
\end{tabular}

${ }^{+} \mathrm{p}<.10 ;{ }^{*} \mathrm{p}<.05 ;{ }^{* *} \mathrm{p}<.01 ;{ }^{* * *} \mathrm{p}<.001$ 
Dado que HLM no ofrece coeficientes estandarizados, en ocasiones no es fácil comparar el impacto relativo de las variables expresadas en escalas y métricas diferentes (variables dicotómicas vs. continuas). La Figura 1 intenta paliar esta limitación mostrando el rango de ganancia de las variables de interés en función de los coeficientes estimados en el modelo final. En el caso de la variable dicotómica, los puntos de ganancia sobre el intercepto indican la diferencia entre dos grupos (tener cuaderno o no). En las variables continuas, la longitud de la barra señala la distancia entre los percentiles 10 y 90 de la distribución de frecuencias de cada variable. Los resultados indican que, una vez descontado el efecto de los antecedentes socio-demográficos del alumnado, las familias y el centro, las variables relativas a la OTL presentan un importante margen de mejora. Así, la asistencia a clase del docente y las prácticas de aula predicen ganancias en torno a un $25 \%$ de la desviación típica de la escala, en tanto que el disponer de cuaderno y de un clima de trabajo ordenado en el aula suponen, respectivamente, un $16 \%$ y $11 \%$ de ganancia adicional.

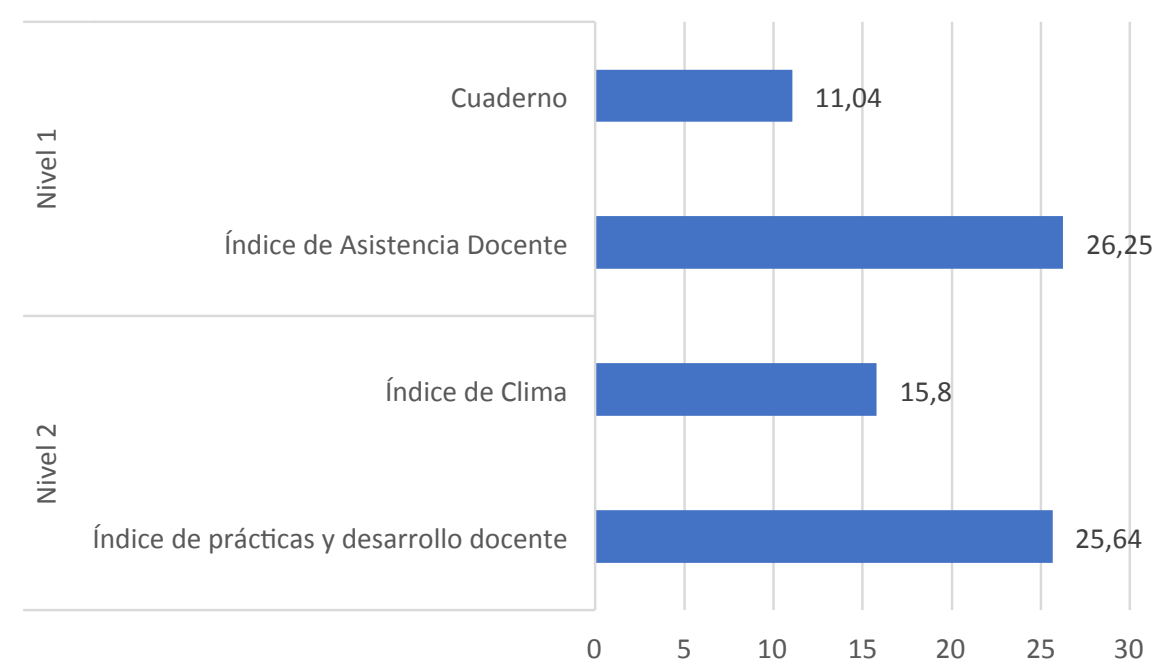

Figura 1. Estimación del efecto de las variables del Modelo del contexto y OTL en el rendimiento en Ciencias Naturales.

\section{Discusión y conclusiones}

Iberoamérica es una de las regiones con mayor desigualdad y donde el tamaño del efecto bruto del centro es bastante alto (Woitschach et al., 2017), por lo que se espera que las variables de contexto tengan un fuerte impacto en los resultados educativos. Los análisis realizados con esta muestra representativa de millones de escolares latinoamericanos así parecen confirmarlo. El modelo I mostró que aproximadamente un 40\% las diferencias en los resultados de los centros se explican por estos factores de contexto o entrada. Los datos señalan un efecto sobre los resultados en Ciencias vinculados al nivel socioeconómico de las familias, la disponibilidad de internet en el hogar y la 
repetición de curso. Por su parte, el SEC del centro está fuertemente vinculado a los resultados, ya que el modelo predice diferencias del orden de 0.92 desviaciones típicas entre el alumnado de los centros con mayor y menor nivel SEC. Esto supone prácticamente un nivel de rendimiento completo en la escala TERCE (UNESCO-OREALC \& LLECE, 2016b). Igualmente, otras características del centro, como titularidad, ruralidad e infraestructuras también están asociadas con los resultados en Ciencias.

En relación al primer objetivo del estudio, el modelo II señala que las variables OTL explican cerca de un $10 \%$ de las diferencias entre los centros y un $2 \%$ de las diferencias entre el alumnado. Son valores más pequeños que los reportados por Angell et al. (2006), pero se encuentran en la línea de los estudios que destacan el poder explicativo de las variables OTL (Lamain et al., 2017; Luyten, 2017; Mo et al., 2013; Scheerens, 2017).

En todo caso, y respondiendo al segundo objetivo, el modelo III muestra que, aún después de controlar los factores de entrada, las variables de interés mantienen su significación estadística, lo que permite concluir que, una vez descontadas las características sociodemográficas, los factores asociados a la OTL mantienen un margen de mejora importante en el funcionamiento de los centros. Además, se advierte que las variables de interés más destacadas son las vinculadas al profesorado, dato que es coherente con los resultados de estudios previos realizados en diferentes países de la región (Cueto et al., 2008, 2014; Martínez-Garrido \& Murillo, 2016; Murillo, 2007; UNESCO-OREALC, \& LLECE, 2000, 2010; Velez et al., 1994). Así, el coeficiente del índice de Prácticas y desarrollo docente, predice que las aulas en las que el profesorado explica con paciencia, anima, felicita, motiva y pregunta con regularidad sobre qué entendió el alumnado, obtendrán en torno a 26 puntos más que las aulas en las que el profesorado puntúa más bajo en este índice. Estos resultados son compatibles con lo señalado por Martínez-Garrido (2015); Torres-Fernández, 2008; UNESCO-OREALC y LLECE (2016a). Por su parte, el índice de Asistencia y puntualidad docente predice ganancias similares puesto que el alumnado cuyo profesorado asiste con regularidad presenta un efecto positivo de 26 puntos en el rendimiento. Estos datos están en línea con las evidencias encontradas por Cueto y Secada (2003); DeStefano, Friedlander, Adelman \& Schuh Moore (2010); Fernández et al. (2016); Hernández-Castilla et al. (2014); Schuh Moore et al. (2012), que señalan la importancia del tiempo efectivo de aula.

Entre las variables de OTL más relevantes se encuentra el Clima de aula que permite aumentar 6 puntos el rendimiento de los estudiantes por cada punto de incremento en el índice, llegando a conseguir las aulas con mejor clima 16 puntos más en su rendimiento. De nuevo los resultados son consistentes con la evidencia disponible sobre la importancia de la atmósfera de trabajo en el aula (Murillo \& Román, 2009; Ramos-Ramírez, 2013; Román, 2010; Torres-Fernández, 2008; UNESCO-OREALC \& LLECE, 2016a).

Finalmente, disponer de un simple cuaderno de clase parece una buena aproximación para una estimación general de los recursos disponibles, puesto que se predicen ganancias en torno al 11\% de la desviación típica después de descontar el efecto de las variables de contexto. Estos datos son coherentes con la evidencia previa que señala que los recursos escolares impactan en los resultados, al menos en los estudios realizados en países en vías de desarrollo (Gaviria et al., 2004; Murillo \& Román, 2009; UNESCO-OREALC \& LLECE, 2016a; Velez et al., 1994). 
Para dar cuenta del tercer objetivo del estudio se comparan los porcentajes de varianza explicados por los modelos I y III, ya que en este último se introducen las variables de interés, una vez que en el primero sólo se consideraron las variables de contexto. En este caso, la ganancia en el porcentaje de varianza entre centros es de 6 puntos porcentuales (38\% en el Modelo I frente al $44 \%$ en el modelo III), lo que puede parecer un efecto modesto, pero que es coincidente con los datos observados en TIMSS (Luyten, 2017). En todo caso la comparación de la varianza explicada por los modelos de ajuste y final permite extraer dos conclusiones generales: en primer lugar, la ecuación que analice la relación OTL-Rendimiento debe incluir variables de ajuste para protegerse de la sobreestimación del efecto de las variables de interés. La segunda lectura tiene que ver con el tamaño del efecto encontrado. Los datos señalan una pequeña ganancia entre los modelos I y III lo que unido al hecho de que las variables de proceso suelen tener mayor impacto en las materias científico-matemáticas que en las áreas de lectoescritura, en las que los resultados parecen estar más influenciados por el contexto familiar del estudiante (Woitschach et al., 2017), podría lleva a concluir que el impacto de las variables OTL es muy modesto. Sin embargo, no conviene olvidar que pequeños efectos sostenidos a lo largo del tiempo pueden marcar diferencias importantes (Prentice \& Miller, 1992), lo que ratifica la relevancia de los resultados encontrados.

Finalmente el estudio tiene algunas limitaciones que conviene tener presente a la hora de interpretar los datos. Las variables OTL manejadas se extrajeron de encuestas de opinión, método que tiene sus limitaciones ya que las respuestas de los informantes pueden estar sometidas a los sesgos propios de la percepción sujetiva y la deseabilidad social (Husen, 1967). Además, en los datos disponibles faltan variables clásicamente vinculadas al constructo OTL, como la cantidad de programa impartido o el tiempo efectivo de aprendizaje, así como registros y observaciones sobre la calidad del proceso instructivo, más allá de las atribuciones realizadas por estudiantes y profesorado (Elliot, 2015). Tampoco se dispuso de una medida de los conocimientos previos del alumnado (más allá de la condición de repetición incluida en este estudio), lo que hubiera permitido controlar el efecto del rendimiento previo del alumnado y de las diferencias en el curriculum en función de esos niveles previos (Floden, 2002), siendo esta, probablemente quitar la razón del pequeño porcentaje de varianza explicada en el nivel 1. Por último, señalar que los modelos empleados son correlacionales por lo que en un futuro sería necesario emplear modelos causales que permitan delimitar la relevancia predictiva de los diferentes componentes del indicador OTL.

\section{Referencias}

Abadzi, H. (2009). Instructional time loss in developing countries: concepts, measurement, and implications. World Bank Research Observer, 24(2), 267-290. doi:10.1093/ wbro/lkp008

Angell, C., Kjærnsli, M., \& Lie, S. (2006). Curricular effects in patterns of student responses to TIMSS science items. En S. J. Howie \& T. Plomp (Eds.), Contexts of learning mathematics and science (pp. 277-290). London: Routledge. 
Bruns \& Luque (2014). Great Teachers How to raise student learning in Latin America and the Caribbean. Washington DC: International bank for recosntruction and development/ The World Bank. doi:10.1596/ 978-1-4648-0151-8.

Carvallo, M. (2006). Factores que afectan el desempeño de los alumnos mexicanos en edad de educación secundaria: un estudio dentro de la corriente de eficacia escolar. Revista Iberoamericana sobre Calidad, Eficacia y Cambio en Educación, 4(3), 30-53. Recuperado de http://www.rinace.net/arts/vol4num3/art3.pdf

Carroll, J. B. (1963). A model of school learning. Teachers College Record, 64, 723-733.

Chaudhury, N., Hammer, J., Kremer, M., Muralidharan, K., \& Rogers, F. H. (2006). Missing in action: Teacher and health worker absence in developing countries. Journal of Economic Perspectives, 20(1), 91-116. doi: 10.1257/089533006776526058

Cueto, S., Guerrero, G., Leon, J., Zapata, M. \& Freire, S. (2014). The relationship between socioeconomic status at age one, opportunities to learn and achievement in mathematics in fourth grade in Peru. Oxford Review of Education, 40(1), 50-72. doi: 10.1080/03054985.2013.873525

Cueto, S., León, J., Ramírez, C., \& Guerrero, G. (2008). Oportunidades de aprendizaje y rendimiento escolar en matemática y lenguaje: resumen de tres estudios en Perú. Revista Iberoamericana sobre Calidad, Eficacia y Cambio en Educación, 6(1), 29-41. Recuperado de https://revistas.uam.es/index.php/reice/article/view/5452/5891

Cueto, S., \& Secada, W. (2003). Eficacia escolar en escuelas bilingues en Puno, Perú. Revista Electrónica Iberoamericana sobre Calidad, Eficacia y Cambio en Educación, 1(1), 1-23. Recuperado de https://goo.gl/2qrMaa

DeStefano, J., Friedlander, E., Adelman, E., \& Schuh Moore, A. (2010). Using opportunity to learn and early grade reading fluency to measure school effectiveness: school quality in Nepal. USAID/ EQUIP2, Washington DC: FHI 360. Recuperado de https://www. epdc.org/sites/default/files/documents/OTL\%20Nepal.pdf

Durlak, J. A., Weissberg, R. P., Dymnicki, A. B., Taylor, R. D., \& Schellinger, K. B. (2011). The impact of enhancing students' social and emotional learning: a metaanalysis of schoolbased universal interventions. Child Development, 82, 405-432. doi:10.1111/j.1467-8624.2010.01564.x

Elliott, S. N. (2015). Measuring opportunity to learn and achievement growth: Key research issues with implications for the effective education of all students. Remedial and Special Education, 36(1), 58-64. doi: 10.1177/0741932514551282

Fernández, T. (2004). De las escuelas eficaces a las reformas educativas de segunda generación. Estudios Sociológicos, 22(65), 377-408. Recuperado de http://tabarefernandez.tripod.com/ee.pdf

Fernández, S., Fernández-Alonso, R., Arias, J. M, Fernández-Raigoso, M., \& Burguera, J. L. (2016). Oportunidad de aprendizaje y eficacia docente. Análisis exploratorio de factores asociados. Bordón, 68(4), 49-65. doi: 10.13042/Bordon.2016.38075

Fernández-Alonso, R., Álvarez-Díaz, M., Suárez-Álvarez, J., \& Muñiz J. (2017). Students' achievement and homework assignment strategies. Frontiers in Psychology, 8, 286. doi: 10.3389/fpsyg.2017.00286

Fernández-Alonso, R. \& Muñiz, J. (2011). Diseño de cuadernillos para la evaluación de las Competencias Básicas. Aula Abierta, 39(2), 3-34. Recuperado de https://dialnet. unirioja.es/servlet/articulo? codigo $=3621228$ 
Fernández-Alonso, R., Suárez-Álvarez, J., \& Muñiz, J. (2012). Imputación de datos perdidos en las evaluaciones diagnósticas educativas. Psicothema, 24(1), 167-175. Recuperado de www.psicothema.com/pdf/3995.pdf

Fernández-Alonso, R., Suarez-Álvarez, J., \& Muñiz, J. (2016). Homework and performance in mathematics: the role of the teacher, the family and the student's background. Revista de Psicodidáctica, 21(1), 5-23. doi:10.1387/RevPsicodidact.13939

Floden, R. E. (2002). The measurement of opportunity to learn. En A. C. Porter \& A. Gamoran (Eds.), Methodological advances in cross-national surveys of educational achievement. Washington: NRC. doi: 10.17226/10322

Gaviria, J. L., Martínez-Arias, R., \& Castro, M. (2004). Un estudio multinivel sobre los factores de eficacia escolar en países en desarrollo: El caso de los recursos en Brasil. Education Policy Analysis Archives, 12(20). Recuperado de https://goo.gl/BhZRZL

Gil Flores, J. (2014). Factores asociados a la brecha regional del rendimiento español en la evaluación PISA. Revista de Investigación Educativa, 32(2), 393-410. doi: 10.6018/ rie.32.2.192441

Gobierno del Principado de Asturias (2011). Evaluación de Diagnóstico Asturias 2010. Oviedo: Consejería de Educación y Ciencia. Recuperado de https://goo.gl/KAv8hN

Hernández-Castilla, R., Murillo, F. J., \& Martínez-Garrido, C. (2014). Factores de ineficacia escolar. Revista Electrónica Iberoamericana sobre Calidad, Eficacia y Cambio en Educación, 12(1), 103-118. Recuperado de https://goo.gl/C4B8ri

Husen, T. (1967). International study of achievement in mathematics: a comparison of twelve countries. (Vol. III). Nueva York: Wiley \& Sons.

Joncas, M., \& Foy, P. (2012). Sample design in TIMSS and PIRLS. En M. O. Martin \& I. V. S. Mullis (Eds.), Methods and Procedures in TIMSS and PIRLS 2011. Chestnut Hill, MA: TIMSS and PIRLS International Study Centre, Boston College.

Klieme, E. (2016). TIMSS 2015 and PISA 2015 How are they related on the country level? Deutsches Institut für Internationale Pädagogische Forschung. Recuperado de: https://goo.gl/1cS1RS

Korpershoek, H., Harms, T., de Boer, H., van Kuijk, M., \& Doolaard, S. (2016). A metaanalysis of the effects of classroom management strategies and classroom management programs on students' academic, behavioral, emotional, and motivational outcomes. Review of Educational Research, 86, 643-680. doi: 10.3102/0034654315626799

Lamain, M., Scheerens, J., \& Noort, P. (2017). Review and "vote count" analysis of OTLeffect studies. En J. Scheerens (Ed.), Opportunity to learn, curriculum alignment and test preparation a research review (pp. 55-101). Switzerland: Springer International Publishing.

Linacre, J. M. (2005). A User's Guide to WINSTEPS/MINISTEP: Rasch-Model Computer Programs (Version 3.55). Chicago: MESA Press.

López, M. (2006). Todo el que llega aquí se contagia: el éxito escolar. En F. J. Murillo (Ed.), Estudios sobre eficacia escolar en Iberoamérica. 15 buenas investigaciones (pp. 261286). Bogotá: Convenio Andrés Bello. Recuperado de https://web.oas.org/childhood/ ES/Lists/Recursos\%20\%20Estudios\%20e\%20Investigaciones/Attachments/21/14.\%20 Estudios\%20sobre\%20eficacia\%20escolar\%20en\%20Iberoamérica.pdf

Liu, X., \& Whitford, M. (2011). Opportunities-to-learn at home: Profiles of students with and without reaching science proficiency. Journal of Science Education and Technology, 20(4), 375-387. doi:10.1007/s10956-010-9259-y 
Luyten, H. (2017). Predictive power of OTL measures in TIMSS and PISA. In J. Scheerens (Ed.), Opportunity to learn, curriculum alignment and test preparation a research review (pp. 103-119). Switzerland: Springer. doi: 10.1007/978-3-319-43110-9_5

McDonnell, L. M. (1995). Opportunity to learn as a research concept and a policy instrument. Educational Evaluation and Policy Analysis, 17(3), 305-322. doi: 10.3102/01623737017003305

Martínez-Garrido, C. (2015). Investigación sobre enseñanza eficaz. Un estudio multinivel para Iberoamerica. (Tesis inédita de doctorado). Universidad Autónoma de Madrid, España.

Martínez-Garrido, C., \& Murillo, F. J. (2016). Investigación Iberoamericana sobre enseñanza eficaz. Revista Mexicana de Investigación Educativa, 21(69), 471-499. Recuperado de http://www.scielo.org.mx/pdf/rmie/v21n69/1405-6666-rmie-21-69-00471.pdf

Marzano, R. J., Marzano, J. S., \& Pickering, D. J. (2003). Classroom management that works. Research-based strategies for every teacher. Alexandria, VA: Association for Supervision and Curriculum Development (ASCD). Recuperado de https://goo.gl/ngMUYR

Mislevy, R. J., Beaton, A., Kaplan, B., \& Sheehan, K. (1992). Estimating population characterstics from sparse matrix samples of item responses. Journal of Education Measurement, 29(2), 131-161. doi:10.1111/j.1745-3984.1992.tb00371.x

Mo, Y., Singh, K. \& Chang, M. (2013) Opportunity to learn and student engagement: a HLM study on eighth grade science achievement. Educational Research for Policy and Practice 12(1), 3-19. doi:10.1007/s10671-011-9126-5

Murillo, F. J. (2007). School effectiveness research in Latin America, En T. Townsend (ed.), International handbook of school effectiveness and improvement, Nueva York: Springer, 75-92.

Murillo, F. J., \& Hernández-Castilla, R. (2011). Factores escolares asociados al desarrollo socio-afectivo en Iberoamérica. Revista Electrónica de Investigación y Evaluación Educativa, 17(2), 1-23. Recuperado de http://www.uv.es/RELIEVE/v17n2/RELIEVEv17n2_2.htm

Murillo, F. J., \& Román, M. (2009). Mejorar el desempeño de los estudiantes de América Latina. Revista Mexicana de Investigación Educativa, 14(41), 451-484. Recuperado de http://www.comie.org.mx/documentos/rmie/v14/n041/pdf/41005.pdf

OECD. (2009). PISA data analysis manual SPSS ${ }^{\circledR}$ (2$^{\mathrm{a}}$ edition). París: OECD Publishing.

OECD (2013). PISA 2012 Assessment and Analytical Framework: Mathematics, Reading, Science, Problem Solving and Financial Literacy. París: OECD Publishing. doi:10.1787/9789264190511-en

Prentice, D. A., \& Miller, D. T. (1992). When small effects are impressive. Psychological Bulletin, 112(1), 160-164. doi:/10.1037/0033-2909.112.1.160

Ramos-Ramírez, G. (2013). La investigación sobre eficacia escolar en el Salvador. Estudio retrospectivo y prospectivo. (Tesis inédita de doctorado), Universidad Autónoma de Madrid, Madrid.

Raudenbush, S., Bryk, A., Cheong, Y. K., Congdon, R., \& du Toit, M. (2011). HLM 7 hierarchical linear and nonlinear modeling. Chicago: SSI Scientific Software International, Inc.

Román, M. (2010). Investigación latinoamericana sobre enseñanza eficaz. Revista Educación y Ciudad, 19, 81-96. Recuperado de https://dialnet.unirioja.es/servlet/ articulo? codigo $=5704988$ 
Scheerens, J. (2016). Educational effectiveness and ineffectiveness a critical review of the knowledge base: Netherlands: Springer. doi: 10.1007/978-94-017-7459-8_1

Scheerens, J. (2017). Meta-analyses and descriptions of illustrative studies. En J. Scheerens (Ed.), Opportunity to learn, curriculum alignment and test preparation. A Research Review (pp. 23-53). Switzerland: Springer International Publishing.

Schuh Moore, A., DeStefano, J., \& Adelman, E. (2012). Opportunity to Learn as a measure of school effectiveness in Guatemala, Honduras, Ethiopia, and Nepal. USAID/ EQUIP2, Washington DC: FHI 360.

Servicio de Evaluación Educativa del Principado de Asturias (2017). ¿Cuánto importa el orden del aula en los resultados educativos? Informes de Evaluación, 7. doi: 10.13140/ RG.2.2.21639.29608

Stallings, J., Knight, S. \& Markham, D. (2014). Using the stallings observation system to investigate time on task in four countries. Washington, DC: World Bank.

Torres-Fernández, P. (2008). La investigación Iberoamericana de eficacia escolar ¿Qué nos dejó a los cubanos? Revista Electrónica Iberoamericana sobre Calidad, Eficacia y Cambio en Educación, 6(4), 81-97. Recuperado de https://revistas.uam.es/index.php/ reice/article/view/5432

UNESCO-OREALC. (2016). Reporte técnico tercer estudio regional comparativo y explicativo. TERCE. Santiago de Chile: UNESCO. Recuperado de http://unesdoc.unesco.org/ images/0024/002471/247123s.pdf

UNESCO-OREALC, \& LLECE. (2000). Primer estudio internacional comparativo sobre lenguaje, matemática y factores asociados, para alumnos del tercer y cuarto grado de la educación básica. Segundo Informe. Santiago de Chile: UNESCO. Recuperado de http:// unesdoc.unesco.org/images/0014/001492/149268s.pdf

UNESCO-OREALC, \& LLECE. (2010). SERCE. Factores asociados al logro cognitivo de los estudiantes de América Latina y el Caribe. Santiago de Chile: UNESCO. Recuperado de http://unesdoc.unesco.org/images/0018/001867/186769S.pdf

UNESCO-OREALC, \& LLECE. (2016a). Informe de resultados del tercer estudio regional comparativo y explicativo. Factores asociados. Santiago de Chile: UNESCO. Recuperado de http://unesdoc.unesco.org/images/0024/002435/243533s.pdf

UNESCO-OREALC, \& LLECE. (2016b). Informe de resultados del tercer estudio regional comparativo y explicativo. Logros de aprendizaje. Santiago de Chile: UNESCO. Recuperado de http://unesdoc.unesco.org/images/0024/002435/243532S.pdf

Velez, E., Schiefelbein, E., \& Valenzuela, J. (1994). Factores que afectan al rendimiento académico en la Educación Primaria. Revisión de la Literatura en América Latina y el Caribe. Revista Latinoamericana de Innovaciones Educativas, 17, 29-53. Recuperado de http://repositorio.minedu.gob.pe/handle/123456789/4317

von Davier, M., Gonzalez, E., \& Mislevy, R. J. (2009). What are plausible values and why are they useful? En M. von Davier \& D. Hastedt (Eds.), IERI monograph series: Issues and methodologies in large-scale assesments 2, 9-36. Princeton, NJ: Educational Testing Service.

Wolff, L., Schiefelbein, E., \& Valenzuela, J. (1994). Mejoramiento de la calidad de la educación primaria en América Latina y el Caribe. Washington, D.C: Banco Mundial. Recuperado de http://www.opech.cl/inv/documentos_trabajo/calidad.pdf 
Woitschach, P., Fernández-Alonso, R., Martínez-Arias, R. y Muñiz, J. (2017). Influencia de los centros escolares sobre el rendimiento académico en Latinoamérica. Revista de Psicología y Educación, 12(2), 138-154. doi: 10.23923/rpye2017.12.152

Fecha de recepción: 24 de octubre de 2017

Fecha de revisión: 31 de octubre de 2017

Fecha de aceptación: 22 de marzo de 2018 